\title{
Lung ultrasound presentation of covid-19 patients: phenotypes and correlations-comment
}

\author{
Galina Nekludova ${ }^{1} \cdot$ Sergey Avdeev ${ }^{1}\left(\mathbb{0} \cdot\right.$ Natalia Trushenko $^{1}$
}

Received: 26 March 2021 / Accepted: 24 May 2021 / Published online: 31 May 2021

(c) Società Italiana di Medicina Interna (SIMI) 2021

Keywords COVID-19 - Lung ultrasound · Acute respiratory distress syndrome

Dear Editor,

There is considerable evidence to date that lung ultrasound (LUS) can be used to assess COVID-19-associated acute respiratory distress syndrome (ARDS). LUS assessment is a semi-quantitative method that assesses the lung zones for the presence of specific artifacts caused by increased extravascular water and/or loss of aeration. LUS can be regarded as a complementary imaging tool that may be used in different settings. However, the predictive value of these LUS findings in COVID-19 patients is not fully known. Therefore, we read with great interest the article by Secco et al [1], published in a recent issue of Internal and Emergency Medicine.

An important finding of this study is that LUS score is related to arterial oxygen partial pressure/ fraction of inspiratory oxygen ratio $(\mathrm{PaO} 2 / \mathrm{FiO} 2)$. That is, the LUS score may reflect changes in lung structure and function in COVID-19 patients, and can also be a signal about the possible need for adequate respiratory assistance. The next important finding of the study by Secco et al is that the severity of LUS impairment predicts 30 day mortality in patients with COVID-19-associated ARDS. In addition, the survival rate in patients with the consolidation pattern was lower than in patients with the interstitial one, although the interstitial and consolidation patterns contribute equally to a decrease in lung aeration.

However, we consider that there are some points to consider for an adequate evaluation of the results. The ability of LUS to predict the need for invasive mechanical ventilation (IMV) and mortality was also demonstrated in several

Sergey Avdeev

serg_avdeev@list.ru

1 Pulmonology Department, I.M. Sechenov First Moscow State Medical University (Sechenov University), 8/2, Trubetskaya Str., 119991 Moscow, Russia studies [2]. However, such results have not been confirmed in other studies. For example, in a multicentre, retrospective, observational study conducted in 6 ICUs in France, the LUS score was not efficient for predicting the 28 day mortality rate in patients with COVID-19 [3]. And, in another study, LUS score had a limited diagnostic value for the identification of patients with the requirement of IMV treatment in a group of patients with moderate to severe COVID-19 infection [4]. How can these inconsistencies between different studies be explained?

COVID-19 pneumonia is a dynamic disease, and the LUS findings are also subject to dynamic changes. The respiratory support (invasive and non-invasive) with high levels of positive end-expiratory pressure may decrease LUS score because of a recruitment effect. LUS score can also be significantly improved by using the prone position in both intubated and non-intubated patients with ARDS. In the previously mentioned study by Seiler et al, respiratory parameters (such as respiratory rate, $\mathrm{SpO} 2 / \mathrm{FiO} 2$, and $\mathrm{ROX}$ index) were stronger predictors than LUS score for identification of patients requiring IMV treatment [4].

Moreover, the requirement of respiratory support and prognosis in COVID-19 could partially depend on patientrelated factors beyond lung pathology identified on LUS. The insufficient sensitivity of LUS as a prognostic tool may be associated with the presence of additional pathological mechanisms leading to hypoxemia. The lungs from patients with COVID-19 showed distinctive vascular features, including severe endothelial damage, widespread thrombosis with microangiopathy. But microvascular thrombosis does not necessarily lead to increased extravascular lung water or decreased lung aeration and, thus, is not quantifiable on LUS [5].

Finally, an important result of the study by Secco et al was the determination of the LUS score threshold (namely, the LUS score $>13$ ) for predicting mortality. However, this 
result is difficult to generalize for widespread use. This is due to both the LUS technique and the scoring system for quantifying the severity of lung injury. To date, there were several studies on LUS for risk stratification that had assessed different numbers of scan areas and had used different systems to assess changes in the lungs. The visualization of B-lines may be dependent on the technical settings, scanners, probes, and frequencies of the ultrasonic pulse. Of course, this is more important when counting the number of B-lines than when using a scoring ultrasound assessment. However, taking into account the technicalities is likely to improve the accuracy and reproducibility of the method. There is no consensus yet regarding the LUS protocols for COVID-19 pneumonia. To summarize the results of different studies, it is necessary to use the standard approaches of the LUS methodology and the scoring system. Therefore, the standardization of LUS protocols for patients with COVID19 is now highly relevant.

Author's contribution All authors contributed equally to the conception, drafting, and final editing of this manuscript.

Funding The authors declared that this letter has received no financial support.

\section{Declarations}

Conflict of interest The authors declare that they have no conflict of interest.
Human and animal rights Not applicable.

Informed consent Not applicable.

\section{References}

1. Secco G, Delorenzo M, Salinaro F et al (2021) Lung ultrasound presentation of COVID-19 patients: phenotypes and correlations. Intern Emerg Med. https://doi.org/10.1007/s11739-020-02620-9

2. Bonadia N, Carnicelli A, Piano A et al (2020) Lung ultrasound findings are associated with mortality and need for intensive care admission in COVID-19 patients evaluated in the emergency department. Ultrasound Med Biol 46(11):2927-2937. https://doi. org/10.1016/j.ultrasmedbio.2020.07.005

3. Duclos G, Bazalguette F, Allaouchiche B et al (2021) Can thoracic ultrasound on admission predict the outcome of critically ill patients with SARS-CoV-2? A French multi-centric ancillary retrospective study. Adv Ther. https://doi.org/10.1007/ s12325-021-01702-0

4. Seiler C, Klingberg C, Hårdstedt M (2021) Lung ultrasound for identification of patients requiring invasive mechanical ventilation in COVID-19. J Ultrasound Med. https://doi.org/10.1002/ jum. 15617

5. Baciarello M, Bonetti A, Vetrugno L et al (2021) Is lung ultrasound score a useful tool to monitoring and handling moderate and severe COVID-19 patients in the general ward? An observational pilot study. J Clin Monit Comput. https://doi.org/10.1007/ s10877-021-00709-w

Publisher's Note Springer Nature remains neutral with regard to jurisdictional claims in published maps and institutional affiliations. 